\title{
INFLUÊNCIA DO TRATAMENTO PÓS-COLHEITA COM CÁLCIO NA CONSERVAÇÃO DE JABUTICABAS ${ }^{1}$
}

\author{
WAGNER FERREIRA DA MOTA², LUIZ CARLOS CHAMHUM SALOMÃO ${ }^{3}$, \\ MARLON CRISTIAN TOLEDO PEREIRA ${ }^{2}$, PAULO ROBERTO CECON ${ }^{4}$
}

\begin{abstract}
RESUMO - A jabuticaba tem grande aceitação para consumo "in natura". Entretanto, é altamente perecível, com pequena conservação pós-colheita. Acredita-se que o cálcio seja capaz de retardar a senescência e manter a firmeza dos frutos. O objetivo do presente trabalho foi de ampliar o período de conservação pós-colheita de jabuticabas, por meio de sua imersão em solução de $\mathrm{CaCl}_{2}$. Os frutos foram colhidos completamente maduros em pomar da UFV, imersos em solução de $\mathrm{CaCl}_{2} 40$ g.L-1 por 0; 5; 10; 20; 40 e 60 minutos e mantidos à temperatura e umidade relativa ambientes por seis dias. Maiores tempos de imersão resultaram em maior retenção de firmeza dos frutos, embora sem afetar a perda de matéria fresca dos mesmos. Houve, em todos os tempos de imersão usados, redução da firmeza e aumento da perda de matéria fresca dos frutos com o aumento do período de armazenagem. A taxa respiratória reduziu-se com o aumento do tempo de imersão. A taxa respiratória também foi decrescente, em todos os tempos de imersão, de 0 a 4 dias após a colheita, aumentando desse dia em diante. A acidez da polpa aumentou durante o armazenamento. Esta reduziu-se suavemente quando os tempos de imersão em $\mathrm{CaCl}_{2}$ variaram de 0 a 20 min e aumentou nos frutos imersos por 40 e $60 \mathrm{~min}$. Apesar dos efeitos significativos obtidos, não houve grande contribuição do cálcio na ampliação do período de comercialização de jabuticabas.
\end{abstract}

Termos para indexação: Myrciaria jaboticaba, armazenamento, firmeza

\section{THE INFLUENCE OF THE POSTHARVEST TREATMENT WITH CALCIUM IN JABUTICABA FRUITS CONSERVATION.}

\begin{abstract}
The jabuticaba fruits has great acceptance for "in natura" consumption. However, it is highly perishable, with short postharvest life. Calcium ions are capable to delay the senescence and to maintain fruit firmness. The objective of this experiment was to increase shelf life of jabuticabas, by means of its immersion in solution of $\mathrm{CaCl}_{2}$. The fruits were harvested fully ripe, immerged in solution of $\mathrm{CaCl}_{2} 40$ g.L. $\mathrm{L}^{-1}$ for $0 ; 5 ; 10 ; 20 ; 40$ and 60 minutes and stored at room temperature and relative humidity for six days. Larger times of immersion resulted in larger retention of fruit firmness, although without affecting its fresh matter loss. In all immersion times there were reduction of firmness and increasing of fresh matter loss of the fruits with increasing of storage period. Longer immersion times reduced respiration rates. Respiration rate reduced, in all immersion times, until the fourth day after the harvest, followed by increasing after the sixth day. The acidity increased during storage and reduced when immersion times in $\mathrm{CaCl}_{2}$ varied from 0 to $20 \mathrm{~min}$ and then increased in the fruits immerged for 40 and $60 \mathrm{~min}$. In spite of the described effects, there was not great contribution of calcium in the extension of commercialization period of the jabuticaba, fruits.
\end{abstract}

Index terms: Myrciaria jaboticaba, storage, fruit firmness

\section{INTRODUÇÃO}

A Jabuticabeira é uma Mirtácea nativa do Brasil e tem como principais espécies Myrciaria cauliflora e M. jaboticaba, sendo a cultivar 'Sabará', da espécie M. jaboticaba, a mais difundida no Brasil (Andersen \& Andersen, 1988). O fruto dessa cultivar é um bacídeo globoso, com 20 a $30 \mathrm{~mm}$ de diâmetro e possui uma polpa macia, esbranquiçada e suculenta, de sabor subácido, que é circundada por um epicarpo fino e com quatro sementes embebidas. Os frutos possuem um pequeno pedicelo e são produzidos em grande quantidade ao longo do tronco ou na axila das folhas que já abscidaram (Wiltbank et al., 1983).

O potencial econômico de comercialização desse fruto é grande em função de suas características organolépticas para consumo "in natura", além de ser utilizado na fabricação de licores e geléias (Magalhães et al., 1996). Entretanto, em função de sua perecibilidade, o período de comercialização pós-colheita é curto, pois há rápida alteração da aparência, decorrente da intensa perda de água, deterioração e fermentação da polpa, observados em apenas dois a três dias após a colheita (Barros et al., 1996).

O cálcio é o nutriente mais freqüentemente associado com a qualidade dos frutos em geral (Sams, 1999). É constituinte natural de parede celular e lamela média dos vegetais. Os seus íons ligam as pectinas, que são formadas por cadeias de ácido poligalacturônico com inserções de ramnose, à parede celular (Kays, 1991). As pontes de cálcio entre os ácidos pécticos ou entre esses e outros polissacarídeos dificultam o acesso e a ação de enzimas pectolíticas produzidas pelo fruto e que causam

\footnotetext{
1 (Trabalho 254/2000). Recebido: 20/11/2000. Aceito para publicação: 11/09/2001.

2 MS, Doutorando em Fitotecnia, DFT - UFV, 36571-000, Viçosa, MG

3 Professor Adjunto, DFT - UFV, 36571-000, Viçosa, MG, e-mail: 1salomao@mail.ufv.br

4 Professor Adjunto, DPI - UFV, 36571-000, Viçosa, MG.
} 
amaciamento, e daquelas produzidas pelos fungos e bactérias que causam deterioração (Conway et al., 1992). É certo que sérias perdas econômicas ocorrem anualmente em órgãos como frutos e hortaliças, devido a desordens fisiológicas e podridões causadas pelo teor inadequado de cálcio em seus tecidos (Shear, 1975).

O íon cálcio, em concentrações adequadas no tecido, tem sido usado para manter a textura e a firmeza, retardar a senescência e reduzir a taxa respiratória e a produção de etileno (Poovaiah, 1986).

A aplicação de cálcio durante o crescimento ou em imersões pós-colheita é usada para aumentar o período de armazenagem de maçãs (Poovaiah, 1986; Lurie \& Klein, 1992), goiabas (Carvalho et al., 1998) e outros frutos. Para a goiaba 'Kumagai', Carvalho et al. (1998) observaram que a imersão em $\mathrm{CaCl}_{2} 40$ g. $\mathrm{L}^{-1}$ a $48^{\circ} \mathrm{C}$, por 5 min, manteve a qualidade do fruto por 28 dias de armazenamento a $10^{\circ} \mathrm{C}$ e $90 \%$ de umidade relativa.

$\mathrm{O}$ presente trabalho objetivou manter a firmeza e, conseqüentemente, ampliar o período de conservação póscolheita de jabuticabas por meio da imersão dos frutos em solução de $\mathrm{CaCl}_{2}$, visando ao consumo "in natura".

\section{MATERIAL E MÉTODOS}

Os frutos de M. jaboticaba Berg. 'Sabará' foram colhidos totalmente maduros no pomar da Universidade Federal de Viçosa, Minas Gerais, em outubro de 1996. Em seguida, foram transportados para o Laboratório de Pós-Colheita, onde foram selecionados e lavados em água potável corrente. Após a lavagem, os frutos foram imersos em solução de hipoclorito de sódio $1 \%$ para desinfestação superficial; e imersos em $\mathrm{CaCl}_{2} 40$ g.L $\mathrm{L}^{-1}$ por $0 ; 5 ; 10 ; 20 ; 40$ e 60 minutos, constituindo os tratamentos. Os frutos de cada tratamento foram, então, acondicionados em grupos de 20 em bandejas rasas de poliestireno, constituindo a unidade experimental. Posteriormente, foram mantidos à temperatura e umidade relativa ambientes, e organizados segundo um delineamento experimental em blocos casualizados em esquema de parcelas subdivididas, tendo nas parcelas os seis tratamentos e nas subparcelas 4,5 ou 6 períodos de amostragem, com quatro repetições.

Foram feitas análises diárias de firmeza do fruto, teor de sólidos solúveis e acidez da polpa, com um total de seis amostragens para firmeza e de cinco para as outras análises. Avaliações da perda de matéria fresca e respiração foram feitas a cada dois dias, totalizando quatro amostragens.

A firmeza foi obtida por uma adaptação da técnica de aplanação (Calbo \& Nery, 1995). Nesta adaptação, utilizou-se um suporte com a base superior côncava para a acomodação do fruto. Sobre o fruto, foi colocado um béquer de peso conhecido com a boca virada para baixo. Em seguida, por meio de um paquímetro, mediu-se o diâmetro da superfície do fruto em contato com o béquer, sendo os resultados expressos em MegaPascal (MPa).

Para a determinação da perda de matéria fresca, as bandejas com os frutos foram pesadas em balança eletrônica e o resultado expresso em porcentagem. A respiração foi determinada por cromatografia gasosa, onde 10 frutos por unidade experimental foram colocados em frasco de um litro e, após uma hora, foi retirada uma alíquota de $1 \mathrm{~mL}$ do ar contido no interior do frasco e injetada em cromatógrafo equipado com coluna de aço inoxidável, empacotada com porapak Q e com detector de condutividade térmica, sendo o resultado expresso $\mathrm{em} \mathrm{mg} \mathrm{CO}{ }_{2} \cdot \mathrm{kg}^{-1} \cdot \mathrm{h}^{-1}$. A determinação dos sólidos solúveis totais foi feita por refratometria, utilizando-se de um refratômetro de mesa, após extração e homogeneização do suco dos frutos de cada bandeja, sendo o resultado expresso em ${ }^{\circ}$ Brix. A acidez titulável foi determinada segundo técnica recomendada pela AOAC (1975), titulando-se $5 \mathrm{~mL}$ de suco do conjunto de frutos de cada bandeja, diluído em água na proporção de $5: 1$, com $\mathrm{NaOH}$ $0,1 \mathrm{~N}$, usando-se fenolftaleína $10 \mathrm{~g} . \mathrm{L}^{-1}$ como indicador. O resultado foi expresso em $\mathrm{g}$ equivalente de ácido cítrico (100 $\mathrm{g}$ de suco $)^{-1}$.

Os resultados foram interpretados por meio de análise de variância e de regressão. Os modelos dos fatores quantitativos foram escolhidos baseados na significância dos coeficientes de regressão, utilizando-se do teste de t de Student, ao nível de 5\% de probabilidade, e no coeficiente de determinação.

\section{RESULTADOS E DISCUSSÃO}

Observou-se redução linear da deformação das jabuticabas com o incremento do tempo de imersão na solução de $\mathrm{CaCl}_{2}$, indicando aumento da firmeza dos frutos (Figura 1). Como o cálcio pode penetrar diretamente através da epiderme e/ ou por fendas naturais da cutícula (Conway et al., 1992), maiores tempos de imersão resultam em maior absorção.

Por outro lado, houve aumento da deformação dos frutos e, portanto, redução da firmeza, com o aumento do período de armazenagem, para todos os períodos de imersão testados. A fase final de desenvolvimento da jabuticaba é caracterizada pelo acentuado decréscimo no teor de pectina, em conseqüência do amadurecimento (Magalhães et al., 1996). Isso explica a redução da firmeza com o período de armazenagem, independentemente do tempo de imersão na solução de cálcio (Figura 1).

O cálcio está associado com a regulação do amadurecimento de frutos, devido à formação de pontes entre os ácidos pécticos e outros ácidos polissacarídeos que atuam como sítios anti-senescência. Essas ligações estabilizam a estrutura da parede e da membrana celular, o que dificulta o acesso e ou interfere na atividade de enzimas responsáveis pelo amaciamento, como a poligalacturonase, mantendo os frutos mais firmes. Apesar da baixa mobilidade no floema (Conway et al., 1992), quanto maior o tempo de imersão, maior será a penetração do íon cálcio, reduzindo a atividade das enzimas responsáveis pelo amaciamento (Conway et al., 1992; Lurie \& Klein, 1992; Conway et al., 1994; Tzoutzoukou \& Bouranis, 1997; Saftner et al., 1998).

Houve leve redução da porcentagem de perda de matéria fresca com o aumento do tempo de imersão em solução de $\mathrm{CaCl}_{2}$ 40 g. $\mathrm{L}^{-1}$ e aumento ao longo do período de armazenagem (Figura 2). $O$ aumento da porcentagem de perda de matéria fresca com o avanço do período de armazenagem deve-se, principalmente, à perda de água por transpiração, em função da rápida deterioração pós-colheita da jabuticaba. Soma-se a isso o fato de o experimento ter sido montado em condições ambientes e o processo de senescência iniciado com o amadurecimento dos frutos, que aceleram os processos respiratório e de perda de água. Menos 


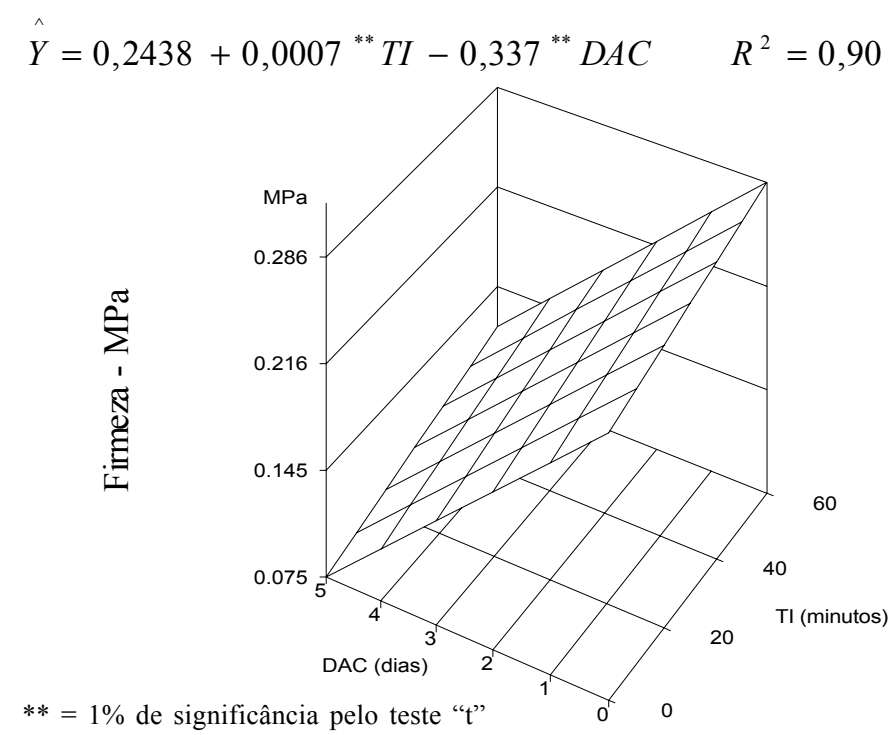

FIGURA 1 - Estimativa da firmeza, em MPa, de jabuticabas armazenadas à temperatura e umidade relativa ambientes, em função dos dias após a colheita (DAC) e após a imersão em solução de $\mathrm{CaCl}_{2}$, nos tempos de $0 ; 5 ; 10 ; 20 ; 40$ e 60 minutos (TI).

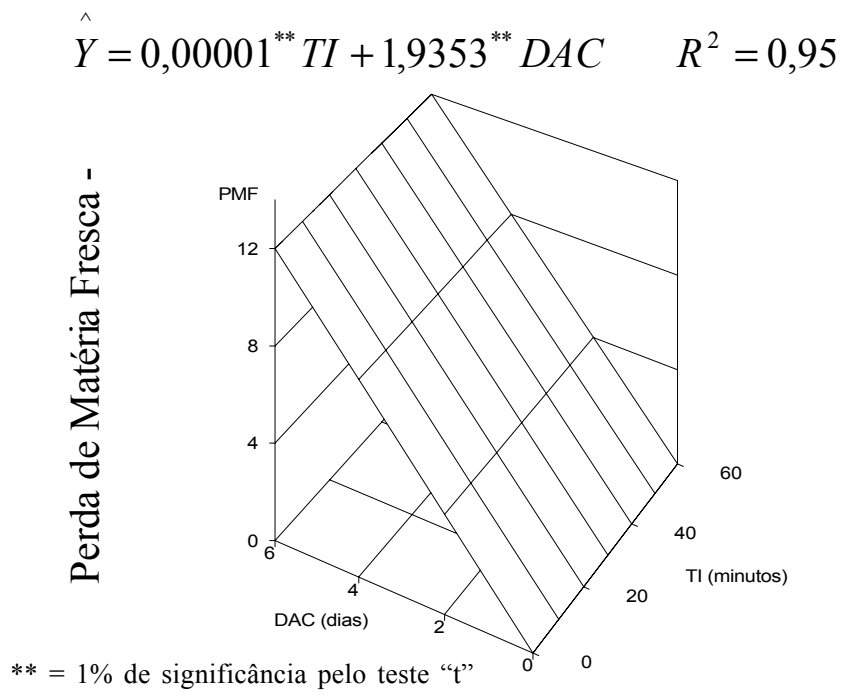

FIGURA 2 - Estimativa da porcentagem de perda de matéria fresca de jabuticabas armazenadas à temperatura e umidade relativa ambientes, em função dos dias após a colheita (DAC) e após a imersão em solução de $\mathrm{CaCl}_{2}$, nos tempos de $0 ; 5 ; 10 ; 20 ; 40$ e 60 minutos (TI).

importante quantitativamente foi a perda de matéria seca resultante do processo respiratório, uma vez que o teor de sólidos solúveis permaneceu em $13,6^{\circ}$ Brix, independentemente do tempo de imersão e do período de armazenagem. A constância de sólidos solúveis totais com o período de armazenamento também foi observada por Lurie \& Klein (1992) em maçã sob condições ambientes.

No presente experimento, não houve efeito de tempos de imersão na solução com cálcio sobre a taxa respiratória (Figura
$\hat{Y}=116,544-0,0137^{n s} T I-10,7537^{* * *} D A C+1,4272^{* * *} D A C^{2} \quad R^{2}=0,59$

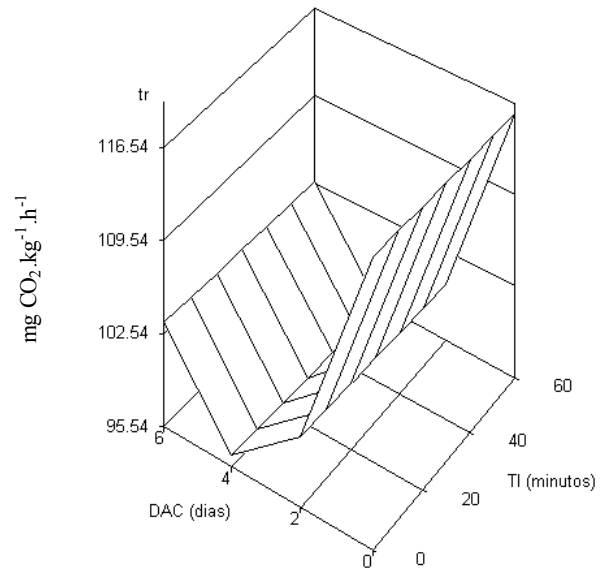

ns = não significativo pelo teste " $\mathrm{t}$ "

$* *$ e $*=1$ e $5 \%$ de significância, respectivamente, pelo teste " $\mathrm{t}$ "

FIGURA 3 - Estimativa da respiração de jabuticabas armazenadas à temperatura e umidade relativa ambientes, em função dos dias após ao colheita e após a imersão em solução de $\mathrm{CaCl}_{2}$, nos tempos de $0 ; 5 ; 10 ; 20$; 40 e 60 minutos.

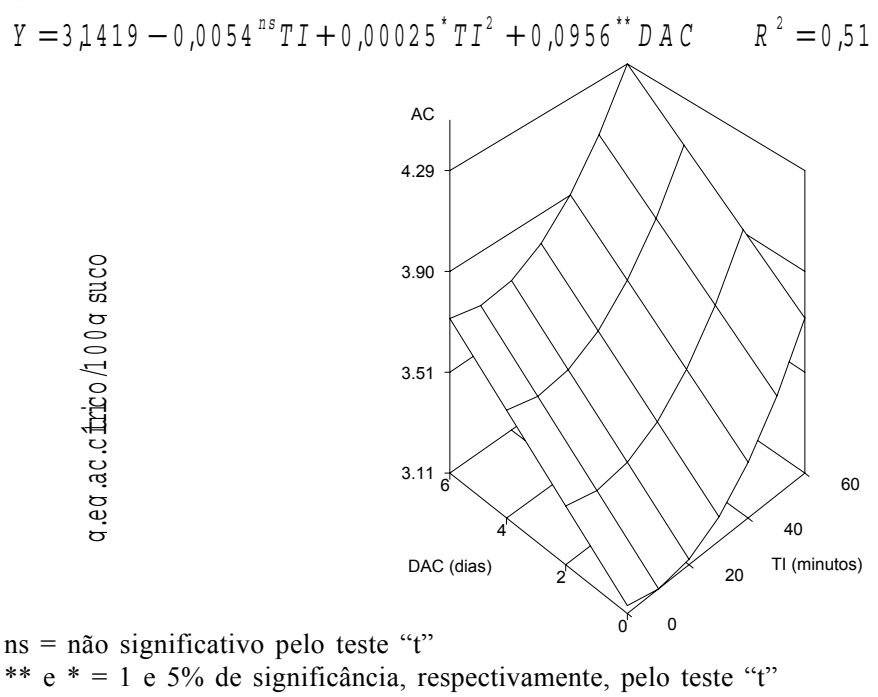

FIGURA 4 - Estimativa da acidez de jabuticabas armazenadas à temperatura e umidade relativa ambientes, em função dos dias após ao colheita (DAC) e após a imersão em solução de $\mathrm{CaCl}_{2}$, nos tempos de $0 ; 5$; $10 ; 20 ; 40$ e 60 minutos (TI).

3). Entretanto, Tzoutzoukou \& Bouranis (1997) observaram redução da taxa respiratória de damascos ao aplicarem cálcio em pré- e pós-colheita. Por outro lado, houve redução da respiração, em todos os tempos de imersão, de 0 a 4 dias após a colheita, seguida de aumento acentuado desse dia em diante (Figura 3), provavelmente pela ocorrência de fermentação na polpa (Barros et al., 1996), uma vez que os frutos foram colhidos totalmente maduros.

A acidez aumentou de forma quadrática em função dos 
tempos de imersão na solução de $\mathrm{CaCl}_{2}$ e linearmente com os períodos de armazenamento (Figura 4). Em todos os tempos de imersão, observou-se que, à medida que se aumentou o período de armazenagem, houve aumento da acidez, provavelmente relacionado com o processo fermentativo, anteriormente discutido.

Houve uma leve redução da acidez no intervalo de tempos de imersão de 0 a 20 minutos, e nos tempos de imersão de 20 a 60 minutos houve acentuado aumento. Altas concentrações de cálcio podem ocasionar dano em frutos devido ao estresse salino (Saftner \& Conway, 1998). Dessa forma, em maiores tempos de imersão, pode ter ocorrido dano, ocasionando plasmólise e conseqüente dano irreversível da membrana, o que pode ter ocasionado a liberação de ácidos.

\section{CONCLUSÃO}

Maiores tempos de imersão de jabuticabas em $\mathrm{CaCl}_{2} 40$ g.. $\mathrm{L}^{-1}$ resultaram em maior retenção de firmeza dos frutos, embora sem afetar a porcentagem perda de matéria fresca dos mesmos. Houve, em todos os tempos de imersão usados, redução da firmeza, aumento da porcentagem de perda de matéria fresca dos frutos e aumento da acidez com o aumento do período de armazenagem. Verificou-se redução da taxa respiratória com o aumento do tempo de imersão, tendência que foi mantida ao longo do período de armazenagem. Apesar disso, a contribuição do cálcio para o aumento do período de comercialização de jabuticabas foi pequena.

\section{REFERÊNCIAS BIBLIOGRÁFICAS}

ANDERSEN, O.; ANDERSEN, V.U. As Fruteiras Silvestres Brasileiras. Rio de Janeiro: Globo, 1988. 203p.

ASSOCIATION OF AGRICULTURAL CHEMIST - AOAC. Official Methods of Analysis of the Association of Official Analytical Chemistry. Washington D.C., 1975. 1094p.

BARROS, R.S.; FINGER, F.L.; MAGALHÃES, M.M. Changes in non-structural carbohydrates in developing fruit of Myrciaria jaboticaba. Scientia Horticulturae, Amsterdam, v.66, p. 209-215, 1996.

CALBO, A. G.; NERY, A. A. Medida de firmeza em hortaliças pela técnica de aplanação. Horticultura Brasileira, v.12, n.1, p.14-18, 1995.

CARVALHO, H.A.; CHITARRA, M.I.F.; CHITARRA, A.B.;
MENEZES, J.B. Eficiência da concentração de cloreto de cálcio e do tempo de imersão no tratamento pós-colheita de goiaba de polpa branca cv. Kumagai. Revista Brasileira de Fruticultura, Cruz das Almas, v.20, n.3, p.375-81, 1998.

CONWAY, W.S.; SAMS, C.E.; McGUIRE, R.G.; KELMAN, A. Calcium treatment of Apples and Potatoes to reduce postharvest decay. Plant Disease, St. Paul, v.76, n.4, p. 329-334, 1992.

CONWAY, W.S.; SAMS, C.E.; WANG, C.Y.; ABBOTT, J.A. Additive effects of postharvest calcium and heat treatment on reducing decay and maintaining quality in Apples. Journal of the American Society for Horticultural Science, Alexandria, v.119, n.1,p. 49-53, 1994.

KAYS, S.J. Postharvest physiology of perishable plant products. New York: AVI Book, 1991, 532p.

LURIE, S.; KLEIN, J.D. Calcium and heat treatment to improve storability of 'Anna' Apples. HortScience, Alexandria, v.27, n.1, p. 36-39, 1992.

MAGALHÃES, M.M.; BARROS, R.S., FINGER, F.L. Changes in structural carbohydrates in developing fruit of Myrciaria jaboticaba. Scentia Horticulturae, Amsterdam, v.66, p. 17-22, 1996.

POOVAIAH, B.W. Role of calcium in prolonging storage life of fruits and vegetables. Food Technology, Chicago, v.40, p. 86-89, 1986.

SAFTNER, R.A.; CONWAY, W.S. Effects of postharvest calcium and fruit coating treatments on postharvest life, quality maintenance and fruit-surface injury in 'Golden Delicious' Apples. Journal of the American Society for Horticultural Science, Alexandria, v.123, n.2, p. 294-298, 1998.

SAMS, C.E. Preharvest factors affecting postharvest texture. Postharvest Biology and Technology, v.15, p. 249-254, 1999.

SHEAR, C.B. Calcium-related disorders of fruits and vegetables. HortScience, Alexandria, v.10, n.4, p. 361, 1975.

TZOUTZOUKOU; C.G.; BOURANIS; D.L. Effect of preharvest application of calcium on the postharvest physiology of Apricot fruit. Journal of Plant Nutrition, Monticello, v.20, n.2\&3, p. 295309, 1997.

WILTBANK, W.V., CHALFUN, N.N.J., ANDERSEN, O. The jaboticaba in Brazil. Proceedings of the American Society for Horticutural Science, Alexandria, v.27A, p. 57-69, 1983. 